\title{
Double excitations in molecules from ensemble density functionals: theory and approximations
}

\author{
Tim Gould, ${ }^{1}$ Leeor Kronik, ${ }^{2}$ and Stefano Pittalis ${ }^{3}$ \\ ${ }^{1}$ Qld Micro- and Nanotechnology Centre, Griffith University, Nathan, Qld 4111, Australia* \\ ${ }^{2}$ Department of Molecular Chemistry and Materials Science, \\ Weizmann Institute of Science, Rehovoth 7610000, Israel \\ ${ }^{3}$ CNR-Istituto Nanoscienze, Via Campi 213A, I-41125 Modena, Italy
}

\begin{abstract}
Double excitations, which are dominated by a Slater-determinant with both electrons in the highest occupied molecular orbital promoted to the lowest unoccupied orbital(s), pose significant challenges for low-cost electronic structure calculations based on density functional theory (DFT). Here, we demonstrate that recent advances in ensemble DFT [Phys. Rev. Lett. 125, 233001 (2020)], which extend concepts of ground-state DFT to excited states via a rigorous physical framework based on the ensemble fluctuation-dissipation theorem, can be used to shed light on the double excitation problem. We find that the exchange physics of double excitations is reproducible by standard DFT approximations using a linear combination formula, but correlations are more complex. In passing, to analyze correlation, we extend the random-phase approximation to ensembles. We then show, using selected test systems, that standard DFT approximations may be adapted to tackle double excitations based on theoretically motivated simple formulae that employ ensemble extensions of expressions that use the on-top pair density.
\end{abstract}

\section{INTRODUCTION}

Within molecular orbital theory, a double excitation refers to a many-electron state that is dominated by a doubly excited Slater determinant. [1] This definition somewhat depends on the single-particle basis being used as a reference, but it is sufficiently evocative to point out that this type of excitation entails a double challenge for present-day low-cost electronic structure methodologies based on density functional theory (DFT).

DFT $[2,3]$ is exact in principle but almost always approximated in practice using exchange-correlation energy models. Still, sophisticated density functional approximations (DFAs) are sufficiently accurate to power many studies of ground states due to an excellent quality to cost ratio. $[4,5]$ The first challenge for double excitations is that, unlike some triplets which may be regarded as a naturally "constrained" ground state, [6] doubly excited target states must be lowest in energy and orthogonal both to the actual ground state and any lower excited singlet. This places them beyond the reach of ground state functional forms. DFT's time-dependent counterpart [7] (TDDFT) does provide a theoretically rigorous way to deal with excitations. However, the second challenge for double excitations is that their prediction using TDDFT requires a highly non-trivial frequency dependence in the "kernel" DFA, which is extremely difficult to obtain in practice. [8-10] In contrast, ensemble DFT for excited states (EDFT) [11-13] has shown promise on model double excitations [14-17] and as a useful framework for wavefunction-based methodologies. [18-21]

In this manuscript, we employ recent theory advances concerning the structure of exact ensemble function-

\footnotetext{
* t.gould@griffith.edu.au
}

als [22-26] with the goal of building high-quality general purpose ensemble DFA (EDFA) that can predict double excitation energies. Firstly, we show that exact ensemble exchange (' $\mathrm{x}$ ') expressions acquire unexpected forms on double excitations. Importantly, this reveals that exchange can be tackled via straightforward re-use of standard DFAs. Secondly, we extend the random-phase approximation (RPA) to ensembles, to scrutinize limitiations of existing correlation ('c') DFAs and to provide a strategy to re-use them. Thirdly, we put together all the gained insights to generate approximations for the overall ensemble 'Hxc' as a functional of the ensemble particle density and, explicitly, of the corresponding ensemble on-top pair-correlation function. Lastly, we demonstrate that EDFAs based on these results are competitive against prominent wavefunction-based alternatives.

\section{ENSEMBLE DENSITY FUNCTIONAL THEORY}

We begin by listing the singlet states, $\left|S_{k}\right\rangle$, we are interested in: a ground state, $\left|S_{0}\right\rangle$, a singly excited state, $\left|S_{1}\right\rangle$, and a doubly excited state, $\left|S_{2}\right\rangle$. [27] We mix these three states in an ensemble,

$$
\hat{\Gamma}=w_{S_{0}}\left|S_{0}\right\rangle\left\langle S_{0}\left|+w_{S_{1}}\right| S_{1}\right\rangle\left\langle S_{1}\left|+w_{S_{2}}\right| S_{2}\right\rangle\left\langle S_{2}\right| .
$$

whose average energy $\mathcal{E}[v]=\min _{\hat{\Gamma}} \operatorname{Tr}[\hat{\Gamma}(\hat{T}+\hat{W}+\hat{v})]=$ $w_{S_{0}} E_{S_{0}}+w_{S_{1}} E_{S_{1}}+w_{S_{2}} E_{S_{2}}$ can be determined variationally, for any weights obeying $w_{S_{0}} \geq w_{S_{1}} \geq w_{S_{2}} \geq 0$. [11] Here, $\hat{T}$ is the kinetic energy operator, $\hat{W}$ is the electronelectron interaction operator and $\hat{v}=\int v(\boldsymbol{r}) \hat{n}(\boldsymbol{r}) d \boldsymbol{r}$ is the external potential operator defined using the density operator $\hat{n}$. Note that Eq. (1) easily generalizes to ensembles mixing degenerate states, where an essential general condition is to work with totally symmetric en- 
sembles. [28] Note further that single and double excitation energies of the system can be computed by varying the weights, e.g. $E_{S_{2}}=\partial_{w_{S_{2}}} \mathcal{E}$.

Analogous to conventional DFT, we may equivalently write,

$$
\mathcal{E}[n]=\mathcal{T}_{s}[n]+\mathcal{E}_{\mathrm{Hx}}[n]+\mathcal{E}_{\mathrm{c}}[n]+\int n(\boldsymbol{r}) v(\boldsymbol{r}) d \boldsymbol{r},
$$

where $\mathcal{T}_{s}, \mathcal{E}_{\mathrm{Hx}}$ and $\mathcal{E}_{\mathrm{c}}$ are the ensemble Kohn-Sham (KS) kinetic energy, Hartree-exchange ('Hx') and correlation density functionals, respectively. Note, the dependence of the functional on the ensemble weights is implicit in the use the calligraphic letters. In the following we further shorten our notation by dropping the obvious dependence on the ensemble particle density $n$.

As usual, the KS potential is given by

$$
v_{s}(\boldsymbol{r}) \equiv v(\boldsymbol{r})+\frac{\delta \mathcal{E}_{\mathrm{Hx}}}{\delta n(\boldsymbol{r})}+\frac{\delta \mathcal{E}_{\mathrm{c}}}{\delta n(\boldsymbol{r})} .
$$

Because we work with totally symmetric ensembles, $v_{s}$, [eq. (3) or generalizations [29]] has the same symmetry as the external potential, $v$. Thus, the corresponding singleparticle states are not only automatically orthogonal to each other but also symmetry adapted. [28]

Our goal in this work is to devise DFAs for $\mathcal{E}_{\mathrm{Hx}}$ and $\mathcal{E}_{\mathrm{c}}$ that are accurate for double excitations. To this end, let us first recall the ansatz-free definitions of these functionals: [23] $\mathcal{T}_{s}=\mathcal{F}^{0}, \mathcal{E}_{\mathrm{Hx}}=\lim _{\lambda \rightarrow 0^{+}} \frac{\mathcal{F}^{\lambda}-\mathcal{T}_{s}}{\lambda}$, and $\mathcal{E}_{\mathrm{c}}=\mathcal{F}^{1}-\mathcal{T}_{s}-\mathcal{E}_{\mathrm{Hx}}$. [24] Here, we introduced the universal functional, $\mathcal{F}^{\lambda}[n]=\min _{\hat{\Gamma}_{\boldsymbol{w}} \rightarrow n} \operatorname{Tr}\left[\hat{\Gamma}_{\boldsymbol{w}}(\hat{T}+\lambda \hat{W})\right]$, which adiabatically connects the non-interacting $(\lambda=0$, $\left.\left|S_{s, k}\right\rangle\right)$ and interacting $\left(\lambda=1,\left|S_{k}\right\rangle\right)$ limits. [30]

A major advantage of EDFT over DFT is that the ensemble KS states are non-interacting configuration state functions (CSFs) rather than single Slater determinants; [23] i.e., minimal linear combinations of Slater determinants which are symmetry adapted both w.r.t. spin and spatial degrees-of-freedom. [31] Thus, interacting and KS states can be labeled by the same quantum numbers. For our initial example [see eq. (1)] we obtain,

$$
\hat{\Gamma}_{s}=w_{S_{0}}\left|S_{s, 0}\right\rangle\left\langle S_{s, 0}\left|+w_{S_{1}}\right| S_{s, 1}\right\rangle\left\langle S_{s, 1}\left|+w_{S_{2}}\right| S_{s, 2}\right\rangle\left\langle S_{s, 2}\right|
$$

with: $\left|S_{s, 0}\right\rangle:=\left|\left[c^{2}\right] h^{2}\right\rangle$ for the ground state; $\left|S_{s, 1}\right\rangle:=$ $\frac{1}{\sqrt{2}}\left[\left|\left[c^{2}\right] h^{\uparrow} l^{\downarrow}\right\rangle+\left|\left[c^{2}\right] h^{\downarrow} l^{\uparrow}\right\rangle\right]$ for the state produced via a single excitation, $h \rightarrow l$, from the HOMO $(h)$ to the LUMO $(l)$; and $\left|S_{s, 2}\right\rangle:=\left|\left[c^{2}\right] l^{2}\right\rangle$ as the double excitation on $h \rightarrow l$. Here, $\left[c^{2}\right] \equiv 1^{2} \cdots(h-1)^{2}$.

We stress that spin-restricted orbitals, $\phi_{i \uparrow}=\phi_{i \downarrow}$, are a direct consequence of preserving symmetries within the EDFT formalism. [23, 28] They appear because Eq. (3) preserves both spatial and spin symmetries even in states (e.g. doublets or triplets) that are usually treated by unrestricted orbitals (UKS). One may say that a restricted KS (RKS) formalism is used throughout. But it is more appropriate to say that a symmetry-adapted KS formalism is used throughout.

In fact, the symmetry adaptation is even more comprehensive. Not only are the single-particle orbitals spin-orbitals, but the many-particle KS states, $\left|S_{s, k}\right\rangle$, are proper (multi determinant) spin eigenstates (singlets, triplets, etc). Symmetry adaptation is also useful to deal with the structure of the multiplets related to the spatial degrees of freedoms: we use this feature later when we study Be and $\mathrm{BH}$. Thus, the KS states, $\left|S_{s, k}\right\rangle$ have a transparent and direct spectroscopical meaning with well-defined quantum numbers and appropriate degeneracies. The employed symmetry-adapted formalism out-performed both UKS DFT and TDDFT in predicting single excitations. [32] In $\mathrm{O}_{2}$, for example, the error in predicted triplet-singlet energy gaps using symmetryadapted EDFT was reduced by a quarter from a similar UKS-based $\triangle \mathrm{SCF}$ calculation.

We then use eq. (4) to obtain ensemble kinetic and Hartree-exchange energies [23],

$$
\begin{gathered}
\mathcal{T}_{s}=\operatorname{Tr}\left[\hat{\Gamma}_{s} \hat{T}\right]=\sum_{k=0}^{2} w_{S_{k}}\left\langle S_{s, k}|\hat{T}| S_{s, k}\right\rangle, \\
\mathcal{E}_{\mathrm{Hx}}=\operatorname{Tr}\left[\hat{\Gamma}_{s} \hat{W}\right]=\sum_{k=0}^{2} w_{S_{k}}\left\langle S_{s, k}|\hat{W}| S_{s, k}\right\rangle,
\end{gathered}
$$

where both involve sums over the weighted contributions of KS-CSFs. $\mathcal{E}_{\mathrm{Hx}}$ is thereby free from spurious self and ghost interactions. But, it represents a conjoint ' $\mathrm{Hx}$ ', which makes it difficult to reuse standard exchange(' $x$ ')DFAs directly. For example, the PBE0 hybrid functional approximation [33] sets $E_{\mathrm{Hxc}}^{\mathrm{PBE} 0}:=E_{\mathrm{H}}+0.25 E_{\mathrm{x}}^{\mathrm{HF}}+$ $0.75 E_{\mathrm{x}}^{\mathrm{PBE}}+E_{\mathrm{c}}^{\mathrm{PBE}}=E_{\mathrm{Hx}}+0.75\left(E_{\mathrm{x}}^{\mathrm{PBE}}-E_{\mathrm{x}}^{\mathrm{HF}}\right)+E_{\mathrm{c}}^{\mathrm{PBE}} u^{\mathrm{X}-}$ ing Hartree-Fock (HF) exchange, with PBE [34] exchange and correlation DFA.

Recently, a fluctuation-dissipation theorem (FDT) was extended and used to further resolve $\mathcal{E}_{\mathrm{Hx}}:=\mathcal{E}_{\mathrm{H}}^{\mathrm{FDT}}+\mathcal{E}_{\mathrm{x}}^{\mathrm{FDT}}$ into ' $\mathrm{H}$ ' and ' $\mathrm{x}$ ' energy expressions that are compatible with existing DFAs. [25] The same extension also offers insights into correlations, discussed further below. The key arguments of Ref. 25 may be summarized as follows: i) DFAs work because they capture universal and essential features of pair correlations; ii) one way to "ensemblize" these pair correlations from first principles is via the density-density response function $\chi$ of the ensemble; iii) the KS response, $\chi_{s}$, allows us to define $\mathcal{E}_{\mathrm{x}}^{\mathrm{FDT}}$ in a way which is formally consistent with existing ' $\mathrm{x}$ '-DFA. $\mathcal{E}_{\mathrm{H}}^{\mathrm{FDT}}$ then includes all the remaining CSF terms; while state-driven correlation [24] can be handled by relating the KS to the interacting response function.

\section{APPROXIMATIONS FOR EXCHANGE ENERGIES}

Using the above principles, we obtain $\mathcal{E}_{\mathrm{H}}^{\mathrm{FDT}}:=$ $\sum_{k k^{\prime}} \min \left(w_{S_{k}}, w_{S_{k^{\prime}}}\right) \int \frac{d \boldsymbol{r} d \boldsymbol{r}^{\prime}}{2\left|\boldsymbol{r}-\boldsymbol{r}^{\prime}\right|} n_{s}^{S_{k} S_{k^{\prime}}}(\boldsymbol{r}) n_{s}^{S_{k^{\prime}} S_{k}}\left(\boldsymbol{r}^{\prime}\right) \quad$ with 
$n_{s}^{S_{k} S_{k^{\prime}}}:=\left\langle S_{s, k}|\hat{n}| S_{s, k^{\prime}}\right\rangle$ for the 'H' functional; and

$$
\mathcal{E}_{\mathrm{x}}^{\mathrm{FDT}}:=-\frac{1}{2} \sum_{i j} f_{\max (i, j)}(i j \mid j i)
$$

for ' $\mathrm{x}$ ', where the expression given above is a convenient corrolary of a result from Ref. 25 that is derived in Section I of the supplementary material [35] [SM-I]. Here, $f_{i}=\sum_{k} w_{S_{k}} \theta_{i}^{S_{k}}$ are the average occupations of orbital, $\phi_{i}$, with occupation $\theta_{i}^{S_{k}} \in\{0,1,2\}$ in state $S_{k}$. This gives, $f_{c}=2$ for all core $(c<h)$ orbitals, $f_{h}=2 w_{S_{0}}+w_{S_{1}}$ for the HOMO and $f_{l}=w_{S_{1}}+2 w_{S_{2}}$ for the LUMO. $(i j \mid k l):=\int \frac{d \boldsymbol{r} d \boldsymbol{r}^{\prime}}{\left|\boldsymbol{r}-\boldsymbol{r}^{\prime}\right|} \phi_{i}^{*}(\boldsymbol{r}) \phi_{j}\left(\boldsymbol{r}^{\prime}\right) \phi_{k}(\boldsymbol{r}) \phi_{l}\left(\boldsymbol{r}^{\prime}\right)$.

Therefore, as a first step to work out a useful approximation we may use the disjointed ' $\mathrm{x}$ ' to obtain,

$$
\mathcal{E}_{\mathrm{Hx}}^{\mathrm{PBE} 0}:=\mathcal{E}_{\mathrm{Hx}}+0.75\left(\mathcal{E}_{\mathrm{x}}^{\mathrm{PBE}}-\mathcal{E}_{\mathrm{x}}^{\mathrm{FDT}}\right) .
$$

The details may, of course, be varied to cover other DFA including range-separated hybrids. The next crucial step is to break $\mathcal{E}_{\mathrm{x}}^{\mathrm{FDT}}:=\sum_{k} w_{k} E_{\mathrm{x}, S_{k}}^{\mathrm{FDT}}$ into individual state contributions so that we can replace exact ' $\mathrm{x}$ ' expressions by existing ' $\mathrm{x}$ '-DFA expressions. For this important task, we follow Fromager [26] to rigorously define,

$$
E_{\mathrm{x}, S_{k}}^{\mathrm{FDT}}:=\frac{\partial \mathcal{E}_{\mathrm{x}}^{\mathrm{FDT}}}{\partial w_{S_{k}}}=-\frac{1}{2} \sum_{i j} \theta_{\max (i, j)}^{S_{k}}(i j \mid j i),
$$

where we used $f_{\max (i, j)}=\sum_{k} w_{S_{k}} \theta_{\max (i, j)}^{S_{k}}$ in eq. (7) to derive the second expression. Note that applying the same principle to Eqs. (5) and (6) yields, $T_{s, S_{k}}=\left\langle S_{s, k}|\hat{T}| S_{s, k}\right\rangle=\sum_{i} f_{i} \int \frac{1}{2}\left|\nabla \phi_{i}\right|^{2} d \boldsymbol{r}$ and $E_{\mathrm{Hx}, S_{k}}=$ $\left\langle S_{s, k}|\hat{W}| S_{s, k}\right\rangle$ as expected. We expand on $E_{\mathrm{Hx}, S_{k}}$ below, near Eq. (12).

It follows from (9) that, $E_{\mathrm{x}, S_{0}}^{\mathrm{FDT}}=-\sum_{i, j \in\{c, h\}}(i j \mid j i) \equiv$ $E_{\mathrm{x}, S_{0}}^{\mathrm{HF}}$, adopts its conventional $\mathrm{HF}$ form for the ground state - by construction. [25] Further algebraic manipulation of the above equations [SM-II] yields,

$$
E_{\mathrm{x}, S_{1}}^{\mathrm{FDT}}=E_{\mathrm{x}, T_{0}}^{\mathrm{HF}}, \quad E_{\mathrm{x}, S_{2}}^{\mathrm{FDT}}=2 E_{\mathrm{x}, T_{0}}^{\mathrm{HF}}-E_{\mathrm{x}, S_{0}}^{\mathrm{HF}} \neq E_{\mathrm{x}, S_{2}}^{\mathrm{HF}},
$$

where both are defined in terms of conventional HF ' $\mathrm{x}$ ' energies for lowest energy singlets, $E_{\mathrm{x}, S_{0}}^{\mathrm{HF}}$, and triplets, $E_{\mathrm{x}, T_{0}}^{\mathrm{HF}}=\left\langle\left[c^{2}\right] h^{\uparrow} l^{\uparrow}|\hat{W}|\left[c^{2}\right] h^{\uparrow} l^{\uparrow}\right\rangle=\left\langle\left[c^{2}\right] h^{\downarrow} l^{\downarrow}|\hat{W}|\left[c^{2}\right] h^{\downarrow} l^{\downarrow}\right\rangle=$ $-\frac{1}{2}\left[\sum_{i, j \in\{c, h, l\}}(i j \mid j i)+\sum_{i, j \in\{c\}}(i j \mid j i)\right]$. Importantly, both $E_{\mathrm{x}, S_{0} / T_{0}}$ have existing DFA counterparts. Also note that the corresponding ' $\mathrm{H}$ ' energies, $E_{\mathrm{H}, S_{k}}^{\mathrm{FDT}}:=$ $\partial_{w_{S_{k}}} \mathcal{E}_{\mathrm{H}}^{\mathrm{FDT}}=\partial_{w_{S_{k}}}\left[\sum_{k} w_{S_{k}} E_{\mathrm{H}, S_{k}}^{\mathrm{HF}}+\left(w_{S_{1}}+w_{S_{2}}\right) E_{S T}\right]$, involve $E_{\mathrm{H}, S_{k}}^{\mathrm{HF}}:=\int \frac{d \boldsymbol{r} d \boldsymbol{r}^{\prime}}{\left|\boldsymbol{r}-\boldsymbol{r}^{\prime}\right|} n_{s}^{S_{k} S_{k}}(\boldsymbol{r}) n_{s}^{S_{k} S_{k}}\left(\boldsymbol{r}^{\prime}\right)$ and $E_{S T}:=$ $2(h l \mid l h)$ [SM-II]. Thus, $E_{\mathrm{H}, S_{1} / S_{2}}^{\mathrm{FDT}}=E_{\mathrm{H}, S_{1} / S_{2}}^{\mathrm{HF}}+E_{S T}$, contain the singlet-triplet splitting terms, $E_{S T}$, that are absent in $E_{\mathrm{x}, S_{1} / S_{2}}^{\mathrm{FDT}}$ but required by $E_{\mathrm{Hx}, S_{k}}=$ $\left\langle S_{s, k}|\hat{W}| S_{s, k}\right\rangle=E_{\mathrm{H}, S_{k}}^{\mathrm{FDT}}+E_{\mathrm{x}, S_{k}}^{\mathrm{FDT}}$.

Eq. (10) states two key results of this work. The first expression shows that single excitations have the same
TABLE I. (Pair-)coefficients for $\mathrm{x}$ and $\mathrm{Hx}$ with different degeneracies, $D_{l}$, of the LUMO. Here $f_{h}=2 w_{S_{0}}+w_{S_{1}}$, $f_{l}=\frac{w_{S_{1}}+2 w_{S_{2}}}{D_{l}}$ is equal for all LUMO, and $F_{l h}=F_{h l}$. Note, 'arb.' (arbitrary) means the result applies to any value of $D_{l}$.

\begin{tabular}{ccc|cc|ccc|ccc}
\hline \hline State & $D_{l}$ & Example & $f_{h}$ & $f_{l}$ & $F_{h h}^{J}$ & $F_{h l}^{J}$ & $F_{l l}^{J}$ & $F_{h h}^{K}$ & $F_{h l}^{K}$ & $F_{l l}^{K}$ \\
\hline$S_{0}$ & arb. & all mol. & 2 & 0 & 4 & 0 & 0 & -2 & 0 & 0 \\
$S_{1}$ & arb. & all mol. & 1 & $\frac{1}{D_{l}}$ & 1 & $f_{l}$ & $f_{l}$ & -1 & $f_{l}$ & $-f_{l}$ \\
$S_{2}$ & 1 & $\mathrm{CH}_{2}$, nit. & 0 & 2 & 0 & 0 & 4 & 0 & 0 & -2 \\
$S_{2}$ & 2 & $\mathrm{BH}$ & 0 & 1 & 0 & 0 & $\frac{1}{2}$ & 0 & 0 & 0 \\
$S_{2}$ & 3 & $\mathrm{Be}$ & 0 & $\frac{2}{3}$ & 0 & 0 & $\frac{1}{5}$ & 0 & 0 & $\frac{1}{15}$ \\
\hline \hline
\end{tabular}

exchange formula, regardless of spin character, $\left(S_{1}\right.$ vs any of $T_{0}$ ) - this result was previously used successfully, [32] but without derivation. The second expression is specific to this work and highlights the importance of ansatz-free procedures. Despite $\left|S_{2}\right\rangle$ being a single Slater determinant, the FDT ' $\mathrm{x}$ ' of the lowest double excitation is not the same as HF ' $x$ ' energy. Rather, it is equivalent to the ground state $\mathrm{HF}$ ' $\mathrm{x}$ ' of twice a triplet minus a singlet.

Finally, using eq. (10) in eq. (8) yields $\mathcal{E}_{\mathrm{Hx}}^{\mathrm{DFA}}:=$ $\mathcal{E}_{\mathrm{Hx}}+\bar{\alpha}\left[\left(w_{S_{0}}-w_{S_{2}}\right)\left(E_{\mathrm{x}, S_{0}}^{\mathrm{DFA}}-E_{\mathrm{x}, S_{0}}^{\mathrm{HF}}\right)+\left(w_{S_{1}}+2 w_{S_{2}}\right)\left(E_{\mathrm{x}, T_{0}}^{\mathrm{DFA}}-\right.\right.$ $\left.\left.E_{\mathrm{x}, T_{0}}^{\mathrm{HF}}\right)\right]$ for the 'Hx' part of general hybrids [SM-II], where $\bar{\alpha}=1-\alpha$ is the complement to the Fock exchange fraction, $\alpha$. The above analysis means we can now reuse any existing $E_{\mathrm{x}, S_{0} / T_{0}}^{\mathrm{DFA}}$ in $\mathcal{E}_{\mathrm{Hx}}^{\mathrm{DFA}}$. Previous work on single excitations successfully assumed [32] that $E_{\mathrm{c}, S_{k}}^{\mathrm{DFA}}$ and $E_{\mathrm{x}, S_{k}}^{\mathrm{DFA}}$ obey the same combination laws. If we extend this to double excitations, we then obtain an 'Hxc' functional,

$$
\begin{aligned}
\mathcal{E}_{\mathrm{Hxc}}^{\mathrm{DFA}} & :=\mathcal{E}_{\mathrm{Hx}}+\left(w_{S_{0}}-w_{S_{2}}\right)\left[\bar{\alpha}\left(E_{\mathrm{x}, S_{0}}^{\mathrm{DFA}}-E_{\mathrm{x}, S_{0}}^{\mathrm{HF}}\right)+E_{\mathrm{c}, S_{0}}^{\mathrm{DFA}}\right] \\
& +\left(w_{S_{1}}+2 w_{S_{2}}\right)\left[\bar{\alpha}\left(E_{\mathrm{x}, T_{0}}^{\mathrm{DFA}}-E_{\mathrm{x}, T_{0}}^{\mathrm{HF}}\right)+E_{\mathrm{c}, T_{0}}^{\mathrm{DFA}}\right] .(11)
\end{aligned}
$$

We revisit the extension below.

The above results can also be generalized to systems with $D_{l}$-fold degenerate LUMOs, such as Be, using ensembles of the form $\hat{\Gamma}=w_{S_{0}}\left|S_{0}\right\rangle\left\langle S_{0}\right|+w_{S_{1}} \hat{\Gamma}_{S_{1}}+w_{S_{2}} \hat{\Gamma}_{S_{2}}$, where $\hat{\Gamma}_{S_{1}}$ and $\hat{\Gamma}_{S_{2}}$ are themselves ensemble over an equal mixture of all degenerate excited states, to yield densities and effective potentials that preserve fundamental spatial symmetries. [28] In general,

$$
E_{\mathrm{Hx}, S_{k}}:=\frac{1}{2} \sum_{i j}\left[F_{i j}^{J, S_{k}}(i j \mid i j)+F_{i j}^{K, S_{k}}(i j \mid j i)\right],
$$

and $E_{\mathrm{x}, S_{k}}^{\mathrm{FDT}}:=-\frac{1}{2} \sum_{i j} f_{\max (i, j)}^{S_{k}}(i j \mid j i)$, where $F_{i j}^{J}=f_{i} f_{j}$ and $F_{i j}^{K}=-\frac{1}{2} F_{i j}^{J}$ except for pairs, $h h, h l_{q}, l_{q} h, l_{q} l_{q^{\prime}}$, involving the HOMO, $h$, and/or one of the LUMOs, $l_{q}$. Values for $F^{J / K, S_{k}}$ and $f^{S_{k}}$ are reported in Table I and derived in SM-II.

Although eq. (11) is only slightly more complex than its ground state equivalent, evaluating it self-consistently for real molecular systems is much more difficult than the ground state problem, for practical reasons outlined in Ref. 29, and because of errors introduced by densitydriven correlations, [24] which come from a failure to 


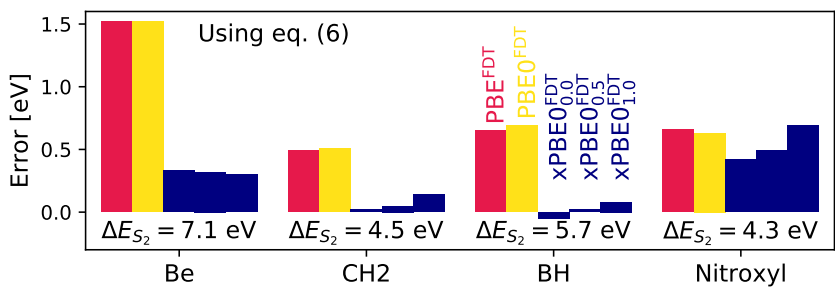

FIG. 1. Errors in excitation energy gaps (in eV) for the double excitation of Be, $\mathrm{CH}_{2}, \mathrm{BH}$ and nitroxyl computed using FDTderived PBE energy expressions [eq. (11)] with (PBE/PBE0, red/yellow) and without ( $\mathrm{xPBE}_{\alpha}$, navy) $\mathrm{PBE}$ correlations included. Reference values are provided as text.

properly account for differences between KS and interacting excited state densities. To obtain the present results, we: i) set $w_{S_{1}}=0$ in all calculations to restrict to mixtures of ground and doubly excited states only; [36] ii) optimize orbitals (mostly) via minimization over unitary transformations; [29] iii) Use the extrapolation procedure described in Ref. [32] to approximately remove densitydriven correlation errors [24]. Full details in SM-III.

Figure 1 shows errors, $\Delta E_{S_{2}}^{\mathrm{EDFA}}-\Delta E_{S_{2}}^{\mathrm{ref}}$ in the resulting EDFA double excitation energies, $\Delta E_{S_{2}}:=E_{S_{2}}-E_{S_{0}}$, of $\mathrm{Be}$ (where $S_{2}$ is five-fold degenerate), $\mathrm{CH}_{2}, \mathrm{BH}$ (two-fold degenerate) and nitroxyl (ONH). Reference data is from Ref. 1 (Be and nitroxyl) and FCI results $\left(\mathrm{CH}_{2}\right.$ and $\left.\mathrm{BH}\right)$ computed for this work. Energies are evaluated using eq. (11) for PBE [34] (red, $\alpha=0$ ) and PBE0 [33] (yellow, $\alpha=0.25)$. Tests using other functionals [37, 38] are of slightly worse quality. Further technical details are given in SM-III. Despite the rigorous theory behind the ' $\mathrm{Hx}$ ' components in eq. (11), the results are uninspiring. This stands in contrast to the success of similar ensemblized DFA in predicting triplet and single excitations [32].

To understand why energies are so poor we first perform x-only PBE0-like (labeled $\mathrm{xPBE}_{\alpha}$ and defined via eq. (11) with $E_{\mathrm{c}}=0$, navy) calculations to investigate the contribution of exchange to the error. Two points are notable: 1) x-only FDT results depend only weakly on $\alpha$, and the variation is larger with stronger correlations, which suggests that exchange physics is treated correctly at the density functional level. 2) Ensemble Hartree-Fock (HF) theory $\left(\mathrm{xPBE}_{1}\right)$ is rather accurate for $\mathrm{Be}, \mathrm{CH}_{2}$ and $\mathrm{BH}$, suggesting that correlations cancel out in these systems, unlike in the DFA. Nitroxyl has strongly correlated ground and excited states so both failures and a wider variation of $\mathrm{XPBE}$ are expected. We therefore conclude that $\mathrm{x}$-DFA (here, PBE) are reliable, and that the error comes primarily from the correlation functional.

\section{APPROXIMATIONS FOR CORRELATION ENERGIES}

As a natural next step, we seek to replicate the success of $\mathcal{E}_{\mathrm{x}}^{\mathrm{FDT}}$ by using the FDT [39] to tackle $\mathcal{E}_{\mathrm{c}}$. Exact eval- uation is impossible in general, so we instead invoke the random-phase approximation (RPA), [39-43] that has found widespread success in modelling difficult ground states; [41-43] and in providing constraints for popular DFAs for correlation. [44, 45] We ensemblize the RPA by using the same KS ensemble density-density response function used to derive eq. (7), [25]

$$
\begin{aligned}
\hat{\chi}_{s}(i \omega) & =\sum_{i j} \frac{\left(f_{i}-f_{j}\right)\left(\epsilon_{i}-\epsilon_{j}\right)}{\left(\epsilon_{i}-\epsilon_{j}\right)^{2}+\omega^{2}} \hat{\rho}_{i} \hat{\rho}_{j}, \\
\mathcal{E}_{\mathrm{c}}^{\mathrm{RPA}} & :=\int_{0}^{\infty} \frac{d \omega}{2 \pi} \operatorname{Tr}\left[\log \left(1-\hat{\chi}_{s} \hat{W}\right)+\hat{\chi}_{s} \hat{W}\right] .
\end{aligned}
$$

Eq. (14) thereby inherits an explicit dependence on the ensemble via the occupation factors, $\left\{f_{i}\right\}$. Here, $\epsilon_{i}$ is the KS eigenvalue of orbital, $i$; Tr and log indicate operator trace and logarithm; and $\operatorname{Tr}\left[\hat{\rho}_{i} \hat{W} \hat{\rho}_{j}\right]=(i j \mid j i)$. Using (13) in (14) yields well-defined energies for $0 \leq f_{l}=$ $w_{S_{1}}+2 w_{S_{2}} \leq 1$ and $f_{h}=2-f_{l}=2 w_{S_{0}}+w_{S_{1}}$ (note, $f_{l}>1$ leads to negative values in the logarithm). Details of RPA calculations are in SM-IV.

Note that the frequency dependence in eq. (14) is averaged within an integration, rather than appearing pointwise as in the key linear-response TDDFT equations for excitations. This fact, together with explicit inclusion of double excitation effects in the ensemble response function, $\hat{\chi}_{s}$, overcomes memory-related issues [8] by letting us extend the RPA to double excitations, as below.

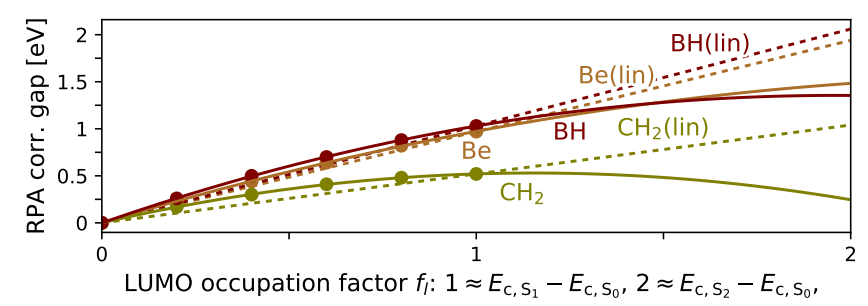

FIG. 2. RPA correlation energy gap, $\mathcal{E}_{\mathrm{c}}^{\mathrm{RPA}}\left(f_{l}\right)-\mathcal{E}_{\mathrm{c}}^{\mathrm{RPA}}(0)$, $[\mathrm{eV}]$ versus $f_{l}$ for $\mathrm{Be}, \mathrm{BH}$ and $\mathrm{CH}_{2}$. Computed values are shown as dots while lines show extrapolated values using the same relationship as exchange (dashed brown, Be; maroon, $\mathrm{BH}$; and olive, $\mathrm{CH}_{2}$ ); and via a quadratic fit (solid lines).

Figure 2 shows the ensemble RPA correlation energies of $\mathrm{Be}, \mathrm{BH}$ and $\mathrm{CH}_{2}$ (dots, we exclude nitroxyl because of its strong correlations) as a function of $f_{l} \leq 1$. However, we are interested in the contribution to the double excitation gap, $E_{\mathrm{c}, S_{2}}-E_{\mathrm{c}, S_{0}} \approx \mathcal{E}_{\mathrm{c}}^{\mathrm{RPA}}\left(f_{l}=2\right)-\mathcal{E}_{\mathrm{c}}^{\mathrm{RPA}}\left(f_{l}=0\right)$, which requires extending results to $f_{l}=2$ - here done by fitting and extrapolating. A linear fit (shown in the figure) between $f_{l}=0$ and $f_{l}=1$ yields, $E_{\mathrm{c}, S_{2}}=$ $2 E_{\mathrm{c}, S_{1}}-E_{\mathrm{c}, S_{0}}$, which we recognise as the correlation energy contribution from eq. (11). However, it also reveals substantial curvature in the computed $\left(0 \leq f_{l} \leq 1\right)$ values. We thus also show a quadratic fit, the simplest model that can capture the curvature, to better extend results to $f_{l}=2$. Self-interaction errors [46-48] in the open shell excitations mean RPA correlation gaps are 
not quantitative. Nonetheless, we do expect RPA to offer useful qualitative insights into the structure of $\mathcal{E}_{\mathrm{c}}$ as weights are varied, e.g., as $w_{S_{2}} \rightarrow 1\left(f_{l} \rightarrow 2\right)$.

The most important revelation of the figure is that the $f_{l}=2$ correlation energy from the (more accurate) quadratic extrapolation is significantly lower than the linear fit in all cases. We therefore expect that at least some of the over-estimation of correlation energies comes from assuming [in eq. (11)] that an exact relationship [eq. (10)] for exchange also applies to correlations, whereas the $R P A$ reveals it does not. That is,

$$
E_{\mathrm{c}, S_{1}}^{\mathrm{DFA}} \approx E_{\mathrm{c}, T_{0}}^{\mathrm{DFA}}, \quad E_{\mathrm{c}, S_{2}}^{\mathrm{DFA}} \not 2 E_{\mathrm{c}, T_{0}}^{\mathrm{DFA}}-E_{\mathrm{c}, S_{0}}^{\mathrm{DFA}},
$$

where $\approx$ indicates that any additional ensemble errors are likely to be similar in magnitude to typical DFA errors, whereas $\not$ indicates that they are likely to be larger.

To improve approximations for correlation energies, we exploit this insight from the RPA. To begin, let us consider a formal device which was used by Becke, Savin and Stoll (BSS) [49] to generate a local density approximation (LDA) that could preserve the degeneracy of multiplets. They replaced the spin polarization, $\zeta=\frac{\left|n_{\uparrow}-n_{\downarrow}\right|}{n}$, in the regular spin-dependent LDA by a function of the on-top (ot) pair density $\zeta \rightarrow \zeta^{\text {ot }}$ (more below) in an otherwise spinless (restricted) theory. Specifically, they used the exact relationship, $\zeta=\zeta^{\mathrm{ot}}:=\sqrt{1-2 P_{\mathrm{Hx}} / n^{2}}$, for a single Slater determinant, where $P_{\mathrm{Hx}}$ is the KS on-top pair density. This result was elegantly further justified in Ref. 50 as a consistent way to escape the symmetry dilemma in spin-unrestricted DFT calculations. Crucially, we show that $\zeta^{\text {ot }}$ can also be exploited to mimic the correction which is required to improve RPA-inspired correlation energies for double excitations.

Next, let us tailor the BSS replacement for ensembles: i) use $P_{\mathrm{Hx}, S_{0}}^{\mathrm{FDT}}=n_{S_{0}}^{2}+P_{\mathrm{x}, S_{0}}^{\mathrm{FDT}}$ in ground states to rewrite $\zeta^{\text {ot }}=\left[-2 P_{\mathrm{x}}^{\mathrm{FDT}} / n^{2}-1\right]^{1 / 2}$ for general states; ii) write eq. (7) as $\int n_{2, \mathrm{x}}^{\mathrm{FDT}}\left(\boldsymbol{r}, \boldsymbol{r}^{\prime}\right) \frac{\boldsymbol{d} \boldsymbol{r} \boldsymbol{d \boldsymbol { r } ^ { \prime }}}{2\left|\boldsymbol{r}-\boldsymbol{r}^{\prime}\right|}$ to obtain $-P_{\mathrm{x}}^{\mathrm{FDT}}(\boldsymbol{r})=$ $-n_{2, \mathrm{x}}^{\mathrm{FDT}}(\boldsymbol{r}, \boldsymbol{r})=\sum_{i j} f_{\max (i, j)} n_{i}(\boldsymbol{r}) n_{j}(\boldsymbol{r})$ where $n_{i}=\left|\phi_{i}\right|^{2}$ is the density of orbital, $i$; iii) obtain,

$$
\zeta^{\mathrm{ot}}(\boldsymbol{r})=\frac{\left[\sum_{i j}\left(2 f_{\max (i, j)}-f_{i} f_{j}\right) n_{i}(\boldsymbol{r}) n_{j}(\boldsymbol{r})\right]^{\frac{1}{2}}}{\sum_{i} f_{i} n_{i}(\boldsymbol{r})} .
$$

By inspection, one sees that (16) reproduces the usual results for non-degenerate ground states, $\left(\zeta^{\mathrm{ot}}=0\right)$ doublets $\left(\zeta_{D_{0}}^{\mathrm{ot}}=\frac{n_{h}}{n}\right)$ and single excitation/triplets $\left(\zeta_{S_{1}}^{\mathrm{ot}}=\zeta_{T_{0}}^{\mathrm{ot}}=\right.$ $\left.\frac{n_{h}+n_{l}}{n}\right)$. Thus, eq. (16) gives "out of the box" DFA results for singular, doublet and triplet ground states. Importantly, and unlike other formulae that are equivalent in ground states, $f_{i} \leq 2$ ensures that eq. (16) is always real so does not require further adjustment to accommodate negative values in the square root, e.g. eq. (11) of Ref. 51.

An additional complication arises in the case of a double excitation between non-degenerate HOMO and LUMO. For regions where the "core" orbital densities are small $\left(n_{c<h} \ll n_{h}, n_{l}\right)$ we obtain, $\zeta_{S_{2}}^{\text {ot }} \approx \sqrt{2 n_{h} / n_{l}}$, by using $f_{h}=0$ and $f_{l}=2$ for the double excitation. When $n_{h}(\boldsymbol{r}) \approx n_{l}(\boldsymbol{r})$ this yields a value of $\zeta^{\text {ot }} \approx \sqrt{2}>1$. A degenerate LUMO can also yield $\zeta^{\text {ot }}>1$ in single excitations. As a final step toward utilizing eq. (16) in approximations we therefore need a way to extend existing DFA to $\zeta>1$. One formula that achieves this is,

$$
\mathcal{E}_{\mathrm{Hxc}}^{\left(\tilde{\zeta}^{\mathrm{ot}}\right)}:=\mathcal{E}_{\mathrm{Hx}}^{\mathrm{DFA}}+\sum_{k=0}^{2} w_{S_{k}} E_{\mathrm{c}, S_{k}}^{\mathrm{DFA}\left(\tilde{\zeta}^{\mathrm{ot}}\right)},
$$

where $E_{\mathrm{c}, S_{k}}^{\mathrm{DFA}\left(\tilde{\zeta}^{\mathrm{ot}}\right)}:=\int d \boldsymbol{r} n_{s}^{S_{k} S_{k}} \epsilon_{\mathrm{c}}^{\mathrm{DFA}}\left(n_{s}^{S_{k} S_{k}}, \nabla n_{s}^{S_{k} S_{k}}, \tilde{\zeta}_{S_{k}}^{\mathrm{ot}}\right)$ uses an heuristic model $\tilde{\zeta}^{\text {ot }}:=\min \left(\zeta^{\mathrm{ot}},\left[\zeta^{\mathrm{ot}}\right]^{-1}\right) \leq 1$ for the effective polarization. Eq. (17) is constructed to reproduce "out of the box" DFA energies for ground states $\left(\zeta^{\mathrm{ot}}=\tilde{\zeta}^{\mathrm{ot}} \leq 1\right)$. Crucially, it also mimics the downward curvature of RPA correlation energies for $f_{l} \rightarrow 2$ $\left(\zeta^{\text {ot }}>1, \tilde{\zeta}^{\text {ot }}<1\right)$ and thus ensures that correlation energies for double excitations become more like $\zeta \rightarrow 0$.

Figure 3 compares results using eq. (17) against results using eq. (11). We see that the new formula denoted $\operatorname{DFA}\left(\tilde{\zeta}^{\text {ot }}\right)$ - substantially improves on eq. (11) in all cases. Excepting Be, Eq. (17) also out-performs both equation-of-motion (EOM) coupled-cluster singles/doubles (CCSD) calculations [52] and CC3 [53]. Thus, the improved model of correlations fixes the most egregious failures of ensemblized DFT and produces reasonable results. Technical details in SM-V.

Before concluding, we briefly address the prototypical "difficult case" of double excitations in dissociating $\mathrm{H}_{2}$ [8-10, 54-56]. Specifically, we study the transition formed by double promotion of the lowest $\sigma$ orbital to the first unoccipied $\sigma$ orbital. These two orbitals are a gerade/ungerade pair: $\phi_{\sigma_{g}} \rightarrow \frac{1}{\sqrt{2}}\left[\phi_{1 s, L}+\phi_{1 s, R}\right]$ and $\phi_{\sigma_{u}} \rightarrow \frac{1}{\sqrt{2}}\left[\phi_{1 s, L}-\phi_{1 s, R}\right]$, where $\rightarrow$ indicates the dissociation (large distance, $D \rightarrow \infty$ ) limit and $\phi_{1 s, L}$ and $\phi_{1 s, R}$ indicate $1 s$ orbitals on the left and right $\mathrm{H}$ atoms. Analytic expressions may be obtained for large $D$ and yield a double excitation gap energy, $\frac{1}{2}-1 / D$ Ha. The dissociation $(D \rightarrow \infty)$ gap is thus $13.6 \mathrm{eV}$.

Figure 4 compares dissociation curves for $\sigma$ states computed using EDFT against exact FCI calculations. It reveals that, like the $\mathrm{H}_{2}$ ground state [25] and despite strong correlations, both $\mathrm{PBE}$ and $\operatorname{PBE}\left(\tilde{\zeta}^{\text {ot }}\right)$ reproduce the correct $-1 / D$ asymptotic behaviour, albeit with an underestimated limit. Inclusion of FDT exchange in PBE0 worsens results by reducing both the effective charge and the asymptotic gap. Ensemble Hartree-Fock theory is even worse, predicting a zero gap for $D \rightarrow \infty$. Full details in SM-VI.

\section{CONCLUSIONS}

In conclusion, we presented a formal and practical ensemble density functional approach to double excitations. First, we showed that a rigorous exact exchange energy expression for double excitations is equivalent 


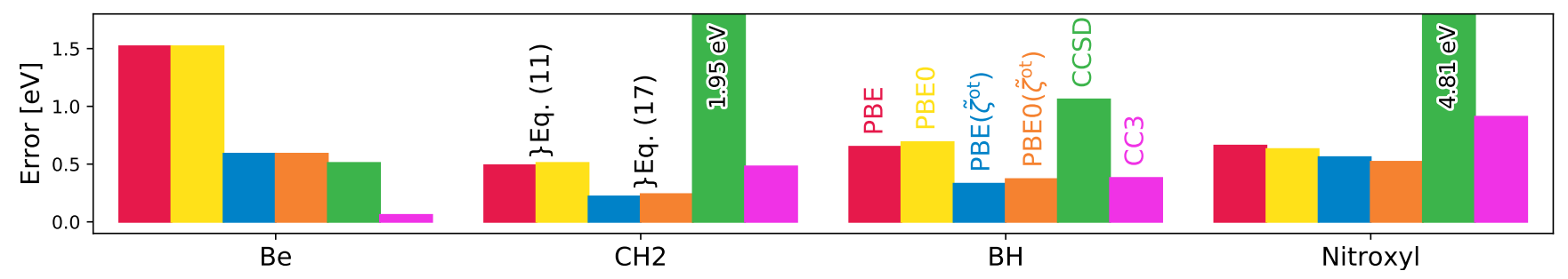

FIG. 3. PBE and PBE0 errors in double excitation energies using eq. (11) (red and yellow, respectively) and eq. (17) (blue and orange). EOM-CCSD (green) and EOM-CC3 (magenta) results are also included for reference. Numbers indicate "off-graph" values.

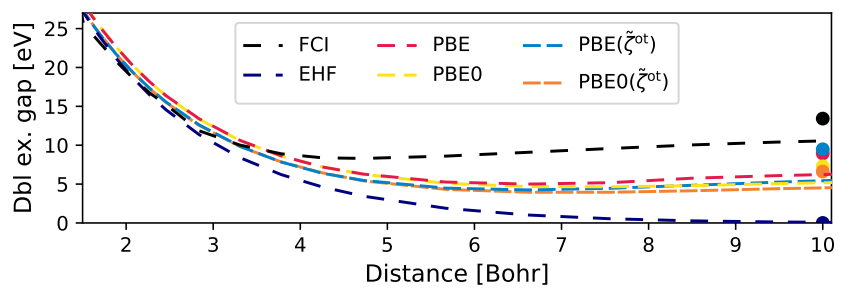

FIG. 4. $\mathrm{H}_{2} \sigma_{g} \rightarrow \sigma_{u}$ double excitation gap using FCI (black), HF (Navy), PBE (red), PBE0 (yellow), $\operatorname{PBE}\left(\tilde{\zeta}^{\text {ot }}\right)$ (blue) and $\operatorname{PBE} 0\left(\tilde{\zeta}^{\mathrm{ot}}\right)$ (orange). Dots show the dissociation gap.

to that of two triplets minus a singlet [eq. (10)] - this counter-intuitive result enables practical reuse of standard exchange-only DFAs. In a second, non-trivial step, we then showed that the corresponding correlation requires additional sophistications. Guided by first principles, we developed an ensemble extension of the RPA expression, which inspired a practical approximation based on the on-top pair-density [eq. (17)] - thus overcoming a natural yet inconsistent guess [eq. (11)].
Results using the EDFAs developed here are already useful for the difficult and varied double excitations studied. Future work should improve our understanding of how on-top pair-densities affect correlations, so that we can devise even better ways to ensemblize conventional DFAs and deal with excited state correlations. Tests should also be extended to larger systems.

Our results do not apply directly to the solid state, due to complexities in their ensemble treatment. They also do not apply directly to systems that are subject to an applied magnetic field or spin-orbit interactions. But, the combination formula follows directly from foundational theories (variational principles [23, 24, 26], group properties [28] and fluctuation-dissipation theorems [25]), which may be extended to a larger class of systems. A first step toward solving these problems would be to derive the necessary analogues of the Gross, Oliveira and Kohn theorems. [11, 12]

\section{ACKNOWLEDGMENTS}

Acknowledgments: TG and LK acknowledge funding from ARC DP200100033. SP acknowledges funding from MIUR PRIN Grant No. 2017RKWTMY.
[1] P.-F. Loos, M. Boggio-Pasqua, A. Scemama, M. Caffarel, and D. Jacquemin, Reference energies for double excitations, J. Chem. Theory Comput. 15, 1939 (2019).

[2] P. Hohenberg and W. Kohn, Inhomogeneous electron gas, Phys. Rev. 136, B864 (1964).

[3] W. Kohn and L. J. Sham, Self-consistent equations including exchange and correlation effects, Phys. Rev. 140, A1133 (1965).

[4] S. Kümmel and L. Kronik, Orbital-dependent density functionals: Theory and applications, Rev. Modern Phys. 80, 3 (2008).

[5] R. O. Jones, Density functional theory: Its origins, rise to prominence, and future, Rev. Modern Phys. 87, 897 (2015).

[6] O. Gunnarsson and B. I. Lundqvist, Exchange and correlation in atoms, molecules, and solids by the spin-densityfunctional formalism, Phys. Rev. B 13, 4274 (1976).

[7] E. Runge and E. K. U. Gross, Density-functional theory for time-dependent systems, Phys. Rev. Lett. 52, 997
(1984).

[8] N. T. Maitra, F. Zhang, R. J. Cave, and K. Burke, Double excitations within time-dependent density functional theory linear response, J. Chem. Phys. 120, 5932 (2004).

[9] P. Romaniello, D. Sangalli, J. A. Berger, F. Sottile, L. G. Molinari, L. Reining, and G. Onida, Double excitations in finite systems, J. Chem. Phys. 130, 044108 (2009).

[10] P. Elliott, S. Goldson, C. Canahui, and N. T. Maitra, Perspectives on double-excitations in TDDFT, Chem. Phys. 391, 110 (2011).

[11] E. K. U. Gross, L. N. Oliveira, and W. Kohn, RayleighRitz variational principle for ensembles of fractionally occupied states, Phys. Rev. A 37, 2805 (1988).

[12] E. K. U. Gross, L. N. Oliveira, and W. Kohn, Densityfunctional theory for ensembles of fractionally occupied states. i. basic formalism, Phys. Rev. A 37, 2809 (1988).

[13] L. N. Oliveira, E. K. U. Gross, and W. Kohn, Densityfunctional theory for ensembles of fractionally occupied states. ii. application to the He atom, Phys. Rev. A 37, 
2821 (1988).

[14] Z.-h. Yang, A. Pribram-Jones, K. Burke, and C. A. Ullrich, Direct extraction of excitation energies from ensemble density-functional theory, Phys. Rev. Lett. 119, 033003 (2017).

[15] F. Sagredo and K. Burke, Accurate double excitations from ensemble density functional calculations, J. Chem. Phys. 149, 134103 (2018).

[16] P.-F. Loos and E. Fromager, A weight-dependent local correlation density-functional approximation for ensembles, J. Chem. Phys, 152, 214101 (2020).

[17] C. Marut, B. Senjean, E. Fromager, and P.-F. Loos, Weight dependence of local exchange-correlation functionals in ensemble density-functional theory: Double excitations in two-electron systems, Faraday Discuss. 224, 402 (2020).

[18] M. Filatov and S. Shaik, A spin-restricted ensemblereferenced Kohn-Sham method and its application to diradicaloid situations, Chem. Phys. Lett. 304, 429 (1999).

[19] M. Filatov, M. Huix-Rotllant, and I. Burghardt, Ensemble density functional theory method correctly describes bond dissociation, excited state electron transfer, and double excitations, J. Chem. Phys. 142, 184104 (2015).

[20] M. Filatov, Spin-restricted ensemble-referenced KohnSham method: Basic principles and application to strongly correlated ground and excited states of molecules, WIREs Comput Mol Sci 5, 146 (2015).

[21] M. Filatov, Ensemble DFT approach to excited states of strongly correlated molecular systems, in DensityFunctional Methods for Excited States, edited by N. Ferré, M. Filatov, and M. Huix-Rotllant (Springer International Publishing, Cham, 2016) pp. 97-124.

[22] T. Gould and J. F. Dobson, The flexible nature of exchange, correlation, and Hartree physics: Resolving "delocalization" errors in a "correlation free" density functional, J. Chem. Phys. 138, 014103 (2013).

[23] T. Gould and S. Pittalis, Hartree and exchange in ensemble density functional theory: Avoiding the nonuniqueness disaster, Phys. Rev. Lett. 119, 243001 (2017).

[24] T. Gould and S. Pittalis, Density-driven correlations in many-electron ensembles: Theory and application for excited states, Phys. Rev. Lett. 123, 016401 (2019).

[25] T. Gould, G. Stefanucci, and S. Pittalis, Ensemble density functional theory: Insight from the fluctuationdissipation theorem, Phys. Rev. Lett. 125, 233001 (2020).

[26] E. Fromager, Individual correlations in ensemble densityfunctional theory: State-driven/density-driven decompositions without additional Kohn-Sham systems, Phys. Rev. Lett. 124, 243001 (2020).

[27] We further assume that no other singly excited state has an energy lower than that of $\left|S_{2}\right\rangle$.

[28] T. Gould and S. Pittalis, Density-driven correlations in ensemble density functional theory: Insights from simple excitations in atoms, Aust. J. Chem. 73, 714 (2020).

[29] T. Gould and L. Kronik, Ensemble generalized KohnSham theory: The good, the bad, and the ugly, J. Chem. Phys. 154, 094125 (2021).

[30] Specifically, minimization is over ensembles, $\hat{\Gamma}_{\boldsymbol{w}}$, with weights, $\boldsymbol{w}$, yielding the target density, $\operatorname{Tr}\left[\hat{\Gamma}_{\boldsymbol{w}} \hat{n}\right]=n$.

[31] The fact that in EDFT KS states must be non-interacting CSFs can be understood via group theory and derived procedurally when evaluating $\mathcal{E}_{\mathrm{Hx}}$ via (degenerate) per- turbation theory - some examples are provided in the Supplementary Material.

[32] T. Gould, Approximately self-consistent ensemble density functional theory: Toward inclusion of all correlations, J. Phys. Chem. Lett. 11, 9907 (2020).

[33] C. Adamo and V. Barone, Toward reliable density functional methods without adjustable parameters: The PBE0 model, J. Chem. Phys. 110, 6158 (1999).

[34] J. P. Perdew, K. Burke, and M. Ernzerhof, Generalized gradient approximation made simple, Phys. Rev. Lett. 77, 3865 (1996).

[35] Supplementary material may be found at XXXXXXXXX,

[36] Technically this breaches ensemble theorems. [11, 12] However, in $\mathrm{BH}$ and Be excluding the single excitation can be rigorously justified on point group symmetry grounds. [28] In $\mathrm{CH}_{2}$ and nitroxyl it is less rigorously justifiable, yet causes no apparent problems for self-consistency.

[37] A. D. Becke, A new mixing of Hartree-Fock and local density-functional theories, J. Chem. Phys. 98, 1372 (1993).

[38] J.-D. Chai and M. Head-Gordon, Systematic optimization of long-range corrected hybrid density functionals, J. Chem. Phys. 128, 084106 (2008).

[39] J. F. Dobson and T. Gould, Calculation of dispersion energies, J Phys : Condens Matter 24, 073201 (2012).

[40] X. Ren, P. Rinke, C. Joas, and M. Scheffler, Randomphase approximation and its applications in computational chemistry and materials science, J Mater Sci 47, 7447 (2012).

[41] H. Eshuis, J. E. Bates, and F. Furche, Electron correlation methods based on the random phase approximation, Theor Chem Acc 131, 1 (2012).

[42] A. Hesselmann, Intermolecular interaction energies from Kohn-Sham random phase approximation correlation methods, in Non-Covalent Interactions in Quantum Chemistry and Physics (Elsevier, 2017) pp. 65-136.

[43] G. P. Chen, V. K. Voora, M. M. Agee, S. G. Balasubramani, and F. Furche, Random-phase approximation methods, Annu. Rev. Phys. Chem. 68, 421 (2017).

[44] J. P. Perdew and Y. Wang, Accurate and simple analytic representation of the electron-gas correlation energy, Phys. Rev. B 45, 13244 (1992).

[45] K. Burke, A. Cancio, T. Gould, and S. Pittalis, Locality of correlation in density functional theory, J. Chem. Phys. 145, 054112 (2016).

[46] F. Furche, Molecular tests of the random phase approximation to the exchange-correlation energy functional, Phys. Rev. B 64, 195120 (2001).

[47] P. Mori-Sánchez, A. J. Cohen, and W. Yang, Failure of the random-phase-approximation correlation energy, Phys. Rev. A 85, 042507 (2012).

[48] T. Gould, A. Ruzsinszky, and J. P. Perdew, Simple selfinteraction correction to random-phase-approximationlike correlation energies, Phys. Rev. A 100, 022515 (2019).

[49] A. Becke, A. Savin, and H. Stoll, Extension of the localspin-density exchange-correlation approximation to multiplet states, Theor. Chim. Acta 91, 147 (1995).

[50] J. P. Perdew, A. Savin, and K. Burke, Escaping the symmetry dilemma through a pair-density interpretation of spin-density functional theory, Phys. Rev. A 51, 4531 (1995). 
[51] G. L. Manni, R. K. Carlson, S. Luo, D. Ma, J. Olsen, D. G. Truhlar, and L. Gagliardi, Multiconfiguration pairdensity functional theory, J. Chem. Theory Comput. 10, 3669 (2014).

[52] J. F. Stanton and R. J. Bartlett, The equation of motion coupled-cluster method. a systematic biorthogonal approach to molecular excitation energies, transition probabilities, and excited state properties, J. Chem. Phys. 98, 7029 (1993).

[53] H. Koch, O. Christiansen, P. Jo/rgensen, A. M. S. de Merás, and T. Helgaker, The CC3 model: An iterative coupled cluster approach including connected triples, J.
Chem. Phys. 106, 1808 (1997).

[54] G. M. J. Barca, A. T. B. Gilbert, and P. M. W. Gill, Hartree-Fock description of excited states of $\mathrm{H}_{2}$, J. Chem. Phys. 141, 111104 (2014).

[55] M. Hellgren, F. Caruso, D. R. Rohr, X. Ren, A. Rubio, M. Scheffler, and P. Rinke, Static correlation and electron localization in molecular dimers from the self-consistent RPA and $G W$ approximation, Phys. Rev. B 91, 165110 (2015).

[56] N. Colonna, M. Hellgren, and S. de Gironcoli, Molecular bonding with the RPAx: From weak dispersion forces to strong correlation, Phys. Rev. B 93, 195108 (2016). 